\title{
The Diversity of Juvenile Sarcoidosis Symptoms
}

\section{Vougiouka O. ${ }^{1}$, Moustaki M. ${ }^{2}$, Nicolaidou P. ${ }^{2}$, Fretzayas A. ${ }^{2}$}

${ }^{1} 2^{\text {nd }}$ Department of Pediatrics, "P and A Kyriakou" Children's Hospital, Athens University School of Medicine, Athens, Greece;

$23^{\text {rd }}$ Department of Pediatrics, “Attikon” University Hospital, Athens University School of Medicine, Athens, Greece

Received December 15, 2011; Accepted June 25, 2012.

Key words: Uveitis - Juvenile sarcoidosis - SACE

Abstract: We report a case of juvenile sarcoidosis, emphasizing the variety of clinical manifestations. The child had uveitis, which is among the most common manifestations of the disease. However, fever of unknown origin, glomerulonephritis and lymphadenopathy were also noticed, underscoring the diversity of the clinical spectrum of the disease.

Mailing Address: Andrew Fretzayas, MD., PhD., $3^{\text {rd }}$ Department of Pediatrics, “Attikon” University Hospital, Athens University School of Medicine, 1 Rimini str., Haidari, 12462 Athens, Greece; Phone: +302 105831 299; Fax: +302 105832 229; e-mail: mar.moustaki@gmail.com 


\section{Introduction}

Juvenile sarcoidosis is a rare, chronic, multisystem, granulomatous disease of obscure etiology. We, herein, report one representative case in an attempt to emphasize the variety of clinical manifestations.

\section{Case report}

A 14-year-old male presented with fever of unknown origin starting fourteen days before admission to the hospital and accompanied with abdominal pain and weight loss. On physical examination there were remarkable paleness and slightly enlarged inguinal lymph nodes. The most prominent laboratory findings were constantly elevated ESR, moderate leucopenia, hyperglobulinemia, inverse CD4/CD8 lymphocyte ratio, low urine specific gravity, mildly compromised renal function and normal values of serum and urine calcium. It was also found skin anergy for tuberculin, candida, tetanus and diphtheria. $67 \mathrm{Ga}$ scan revealed increased uptake by lacrimal and parotid glands (panda pattern, Figure 1). Inguinal lymph node biopsy (Figure 2) was compatible with sarcoidosis while kidney biopsy (Figure 3) showed focal mesangiohyperplastic glomerulonephritis. Chest-X-ray and thoracic computed tomography were both normal as well as pulmonary function tests.

Serum angiotensin converting enzyme (SACE) values were elevated $(87 \mathrm{U} / \mathrm{l})$. Prednisolone at a dose of $0.5 \mathrm{mg} / \mathrm{kg} /$ day $(35 \mathrm{mg}$ daily) was administered for six months and gradually tapered. During his hospitalization he experienced an attack of anterior uveitis which was treated with additional local steroids. Five years later he remains asymptomatic, without any evidence of eye or other organ involvement while SACE values also remain within normal limits.

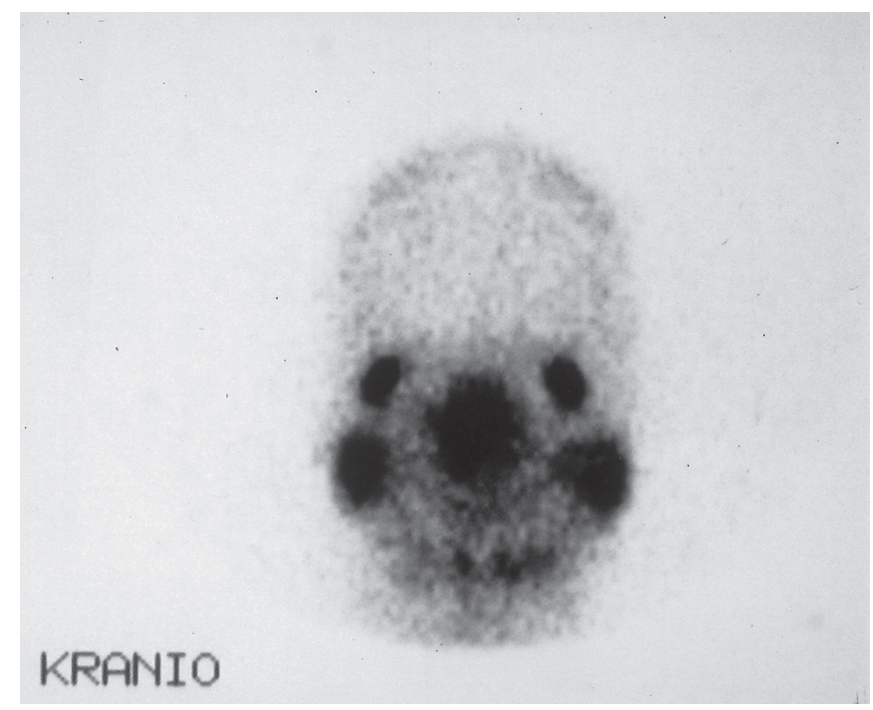

Figure 1 - Panda pattern of isotope uptake on $67 G a$ scan. 


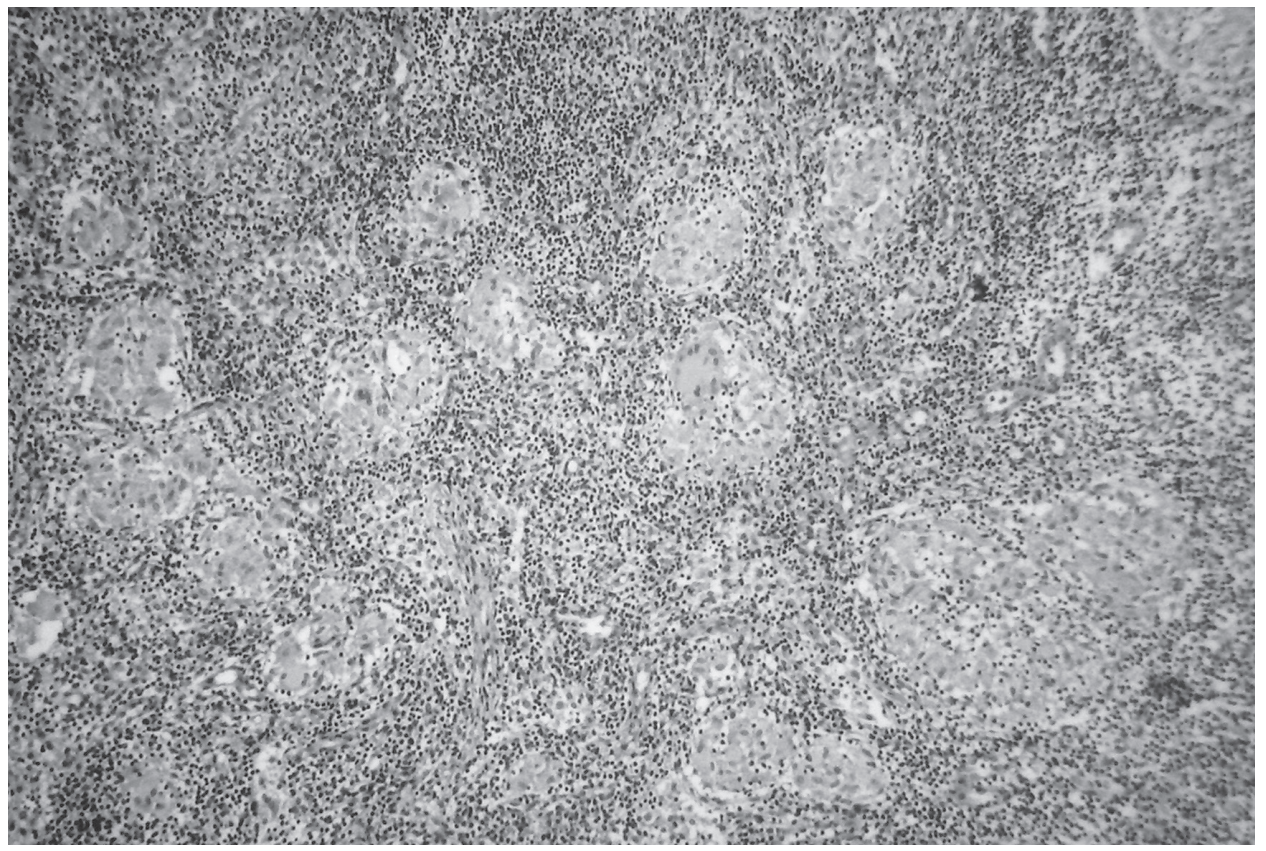

Figure 2 - Granulomatous pattern on inguinal node biopsy.

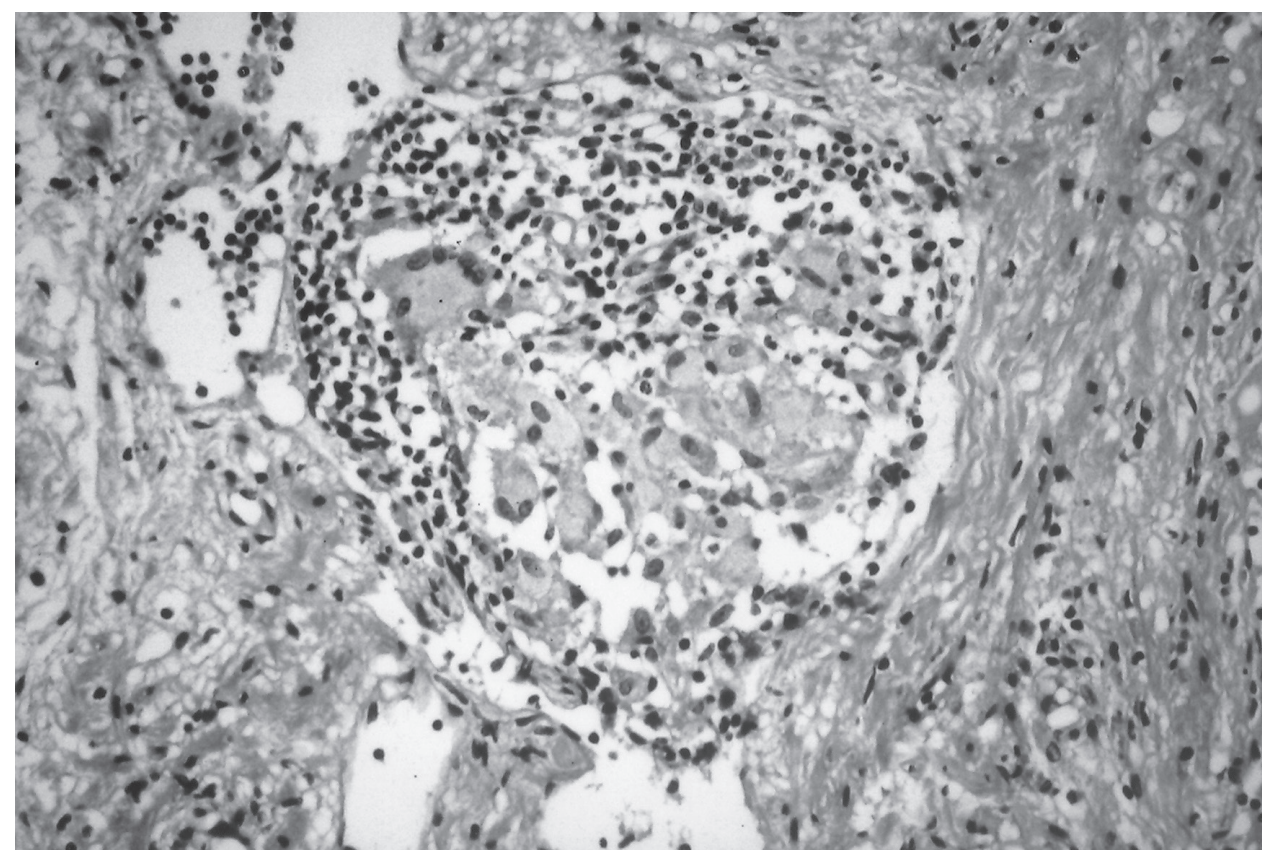

Figure 3 - Focal mesangiohyperplastic glomerulonephritis.

Vougiouka O.; Moustaki M.; Nicolaidou P.; Fretzayas A. 


\section{Discussion}

The exact prevalence, as well as the incidence of sarcoidosis remains speculative due to high frequency of asymptomatic individuals. There is a slightly higher incidence of sarcoidosis in females and the disease is rarely confronted in children (Shetty and Gedalia, 2008).

It has become widely recognized that patients suffering from sarcoidosis are prone to have manifestations related to their age at onset of the disease. In children, during their first four year period of life florid manifestations seem to constitute a separate entity. In older children sarcoidosis presents as a multisystem disorder often accompanied by non specific, constitutional symptoms at onset (Fretzayas et al., 2011). Lung is the most common organ involved at this age group (Fretzayas et al., 2011), although this was not the case with our patient. It should be noted that in a Danish report only $10 \%$ of children with sarcoidosis had normal chest-X-ray (Hoffmann et al., 2004). Fever of unknown origin was the cardinal symptom of our patient indicating that children with this condition should also be investigated for sarcoidosis.

Eye is the second most commonly affected organ, especially in older children. Ophthalmic sarcoidosis is manifested as uveitis with anterior segment involvement in the majority of cases and iris nodules whereas conjuctival granuloma and lacrimal gland involvement are occasionally seen (Laghmari et al., 2008; Herbort et al., 2009).

Although it has been stated that kidneys are affected in $6 \%$ of children, renal function assessed by creatinine clearance determination was found to be compromised in a considerably higher percentage (60\%) (Coutant et al., 1999). Renal involvement has been attributed either to granuloma formation or to nephrocalcinosis resulting from concomitant hypercalciuria, even in the absence of hypercalcemia (Coutant et al., 1999; Shetty and Gedalia, 2008). In our patient kidney biopsy revealed mesangiohyperplastic glomerulonephritis which is uncommon in sarcoidosis as to the best of our knowledge membranous glomerulonephritis was previously described in sarcoidosis (Dimitriades et al., 1999).

In general, in children older than 5 years of age, the organs that are more commonly involved are lungs in more than $90 \%$ (mainly hilar lymphadenopathy), peripheral lymph nodes (40\%), eyes (29-50\%) and skin (25\%) (Clement et al., 2005). Neurosarcoidosis, bone lesions and cardiac involvement are seldom recognized in children. On the contrary neurosarcoidosis is encountered in $5-15 \%$ in adults whereas cardiac involvement is quite high in adults (20-78\%) based on necropsy series. Bone lesions are rarely seen even in adult population with sarcoidosis (Jansen and Geusens, 2005; Moller, 2005).

A positive tissue biopsy from one organ together with compatible clinical and radiographic findings is reasonable for making the diagnosis of sarcoidosis. It should be noted that there is no pathognomonic diagnostic procedure or histological pattern because epithelioid cell granuloma may also be found in tuberculosis, 
leprosy, syphilis, fungal infections, berylliosis and malignancies. However, the initial evaluation of sarcoidosis is based on a detailed history, a thorough clinical examination and a laboratory investigation that should include: chest-X-ray, pulmonary function tests, peripheral blood counts, serum biochemistries, urine analysis, ophthalmologic examination and tuberculin skin test and biopsy from an easily accessible organ such as peripheral lymph node or skin lesion (Hunninghake et al., 1999). In adults fiberoptic bronchoscopy with mucosal biopsy, transbronchial lung biopsy and bronchoalveolar lavage (BAL) is recommended (Hunninghake et al., 1999). However, transbronchial lung biopsy is not in general a feasible procedure in children and therefore it is not recommended.

While SACE believed to be produced by epithelioid cells of sarcoid granuloma, it is elevated in diverse conditions (Shetty and Gedalia, 2008), i.e. miliary tuberculosis, leprosy, Gaucher disease, primary biliary cirrhosis, etc. It is far from to be considered as a pathognomonic diagnostic test. Nevertheless, consecutive determinations of SACE have been shown to be a tool for monitoring treatment efficacy or can be used as an indicator of disease recurrence.

Imaging techniques can contribute considerably to the diagnosis by revealing lung involvement and extrapulmonary manifestations of the disease. Apart from chest-Xray, isotopic studies and high resolution CT scan have been used extensively. Many clinicians perform bronchoalveolar lavage (BAL) in order to recognize alveolitis that may precede granuloma formation in the lungs (Hunninghake et al., 1999). However, in children lymphocytosis which is usually found from the BAL analysis does not correlate with disease activity, treatment response or prognosis (Chadelat et al., 1993). For this reason and given the absence of respiratory symptoms and of abnormal findings in the CT scan we did not perform bronchoscopy in our patient.

On a total body 67Ga-citrate scan the appearance of a "panda pattern" especially when combined with a "lambda pattern" may support greatly the diagnosis (Pattishal et al., 1986). The panda sign showed $74 \%$ sensitivity for the identification of patients with stage I or II sarcoidosis at presentation (Sulavik et al., 1990). However, it is of interest to note that increased isotope uptake may also be seen in lymphoma, Sjogren syndrome and acquired immunodeficiency syndrome (Kurdziel, 2000 ). In one study the panda sign was seen in $4 \%$ (23 of 540) of patients who did not have sarcoidosis (Sulavik et al., 1990).

The differential diagnosis of sarcoidosis is extremely broad (Shetty and Gedalia, 2008; Fretzayas et al., 2011) and includes juvenile idiopathic arthritis, lymphoma, other interstitial lung disease as well as Loefgren's and Mikulicz diseases. Certain infectious entities attributed to mycobacteria and fungi should also be ruled out when granulomatous lesions are found on lung biopsy. In case of hepatic granulomas, cat scratch disease and granulomatous hepatitis should be excluded as well. It should be noted that in children younger than 4 years of age early onset sarcoidosis is quite rare and has a distinct triad of skin, joint and eye disorders without lung involvement (Shetty and Gedalia, 2008; Fretzayas et al., 
2011). This entity should be differentiated from Blau syndrome (rash/arthritis/ ocular symptoms) which is a familial disease transmitted in an autosomal dominant manner (Blau, 1985). As it was shown, both disorders have been linked to Nod2 mutations that cause constitutive NF-KB activation (Kambe et al., 2005).

In conclusion, the clinical spectrum of juvenile sarcoidosis, on initial presentation, varies from common specific manifestations such as uveitis to general symptoms such as fever of unknown origin. For this reason a high index of suspicion is prudent in relevant cases.

\section{References}

Blau, E. B. (1985) Familial granulomatous arthritis, iritis and rash. J. Pediatr. 107, 689-693.

Chadelat, K., Baculard, A., Grimfeld, A., Tournier, G., Boule, M., Boccon-Gibod, L., Clement, A. (1993) Pulmonary sarcoidosis in children: serial evaluation in bronchoalveolar lavage cells during corticosteroid treatment. Pediatr. Pulmonol. 16, 41-47.

Clement, A., Epaud, R., Fauroux, B. (2005) Sarcoidosis in children. Eur. Respir. Monogr. 10, 251-258.

Coutant, R., Leroy, B., Niaudet, P., Loirat, C., Dommergues, J. P., André, J. L. Baculard, A., Bensman, A. (1999) Renal granulomatous sarcoidosis in children: a report of 11 cases and a review of the literature. Eur.J. Pediatr. 158, 154-159.

Dimitriades, C., Shetty, A. K., Vehaskari, M., Craver, R. D., Gedalia, A. (1999) Membranous nephropathy associated with childhood sarcoidosis. Pediatr. Nephrol. 13, 444-447.

Fretzayas, A., Moustaki, M., Vougiouka, O. (2011) The puzzling clinical spectrum and course of juvenile sarcoidosis. World J. Pediatr. 7, 103-110.

Herbort, C. P., Rao, N.A., Mochizuki, M. (2009) International criteria for the diagnosis of ocular sarcoidosis: results of the first International Workshop on Ocular Sarcoidosis (IWOS). Ocul. Immunol. Inflamm. 17, 160-169.

Hoffmann,A. L., Milman, N., Byg, K. E. (2004) Childhood sarcoidosis in Denmark 1979-1994: incidence, clinical features and laboratory results at presentation in 48 children. Acta Paediatr. 93, 30-36.

Hunninghake, G.W., Costabel, U., Ando, M., Baughman, R., Cordier, J. F., du Bois, R., Eklund, A., Kitaichi, M., Lynch, J., Rizzato, G., Rose, C., Selroos, O., Semenzato, G., Sharma, O. P. (1999) ATS/ERS/WASOG statement on sarcoidosis. American Thoracic Society/European Respiratory Society/World Association of Sarcoidosis and other Granulomatous Disorders. Sarcoidosis Vasc. Diffuse Lung Dis. 16, 149-173.

Jansen, T. L. Th. A., Geusens, P. P. M. M. (2005) Sarcoidosis: joint, muscle and bone involvement. Eur. Respir. Monogr. 10, 210-219.

Kambe, N., Nishikomori, R., Kanazawa, N. (2005) The cytosolic pattern-recognition receptor Nod2 and inflammatory granulomatous disorders. J. Dermatol. Sci. 39, 71-80.

Kurdziel, K.A. (2000) The panda sign. Radiology 215, 884-885.

Laghmari, M., Skiker, H., Boutimzine, N., Daoudi, R. (2008) Uveitis during the course of sarcoidosis in a child: difficult diagnosis of a case. Bull. Soc. Belge Ophtalmol. 307, 47-51.

Moller, D. R. (2005) Rare manifestations of sarcoidosis. Eur. Respir. Monogr. 10, 233-250.

Pattishal, E. N., Strope, G. L., Spinola, S. M., Denny, F.W. (1986) Childhood sarcoidosis. J. Pediatr. 108, $169-177$.

Shetty, A. K., Gedalia, A. (2008) Childhood sarcoidosis: A rare but fascinating disorder. Pediatr. Rheumatol. Online J. 6, 16 .

Sulavik, S. B., Spencer, R. P., Weed, D. A., Shapiro, H. R., Shiue, S. T., Castriotta, R. J. (1990) Recognition of distinctive patterns of gallium-67 distribution in sarcoidosis. J. Nucl. Med. 31, 1909-1914. 